\title{
The penetration of Human Defensin 5 (HD5) through bacterial outer membrane: Simulation studies
}

\section{Tadsanee Awang}

Kasetsart University - Bangkhen Campus: Kasetsart University

Prapasiri Pongprayoon ( $\nabla$ fsciprpo@ku.ac.th )

Kasetsart University Faculty of Science https://orcid.org/0000-0002-1472-8241

\section{Research Article}

Keywords: Steered molecular dynamic simulations, Antimicrobial peptides, Human defensin 5, LPS, Molecular dynamics simulations

Posted Date: June 21st, 2021

DOI: https://doi.org/10.21203/rs.3.rs-579559/v1

License: (c) (i) This work is licensed under a Creative Commons Attribution 4.0 International License. Read Full License 


\section{Abstract}

Human a-defensin 5 (HD5) is one of cationic antimicrobial peptides which plays a crucial role in an innate immune system in human body. HD5 shows the killing activity against a broad spectrum of pathogenic bacteria by making a pore in a bacterial membrane and penetrating into a cytosol. Nonetheless, its pore-forming mechanisms remain unclear. Thus, in this work, the constant-velocity steered molecular dynamics (SMD) simulation was used to simulate the permeation of a dimeric HD5 into a gram-negative LPS membrane model. Arginine-rich HD5 is found to strongly interact with a LPS surface. Upon arrival, arginines on HD5 interact with lipid A head groups and then drag these charged moieties down into a hydrophobic core resulting in the formation of water-filled pore. Although all arginines are found to interact with a membrane, R13 and R32 appear to play a dominant role in the HD5 adsorption on a gram-negative membrane. Furthermore, one chain of a dimeric HD5 is required for HD5 adhesion. The interactions of arginine-Lipid A head groups play a major role in adhering a cationic HD5 on a membrane surface and retarding a HD5 passage in the meantime.

\section{Introduction}

Antimicrobial peptides (AMPs) are a class of small peptides that are important for the innate immune system in organisms. AMPs have a wide range of antimicrobial activities against bacteria, fungi, viruses, and parasites [1, 2]. Defensin is one of the main families of AMPs in human. They can be divided into three subfamilies ( $a-, \beta-$, and $\Theta$ - defensins) depending on the regiospecific disulfide-bond linkages [3]. All defensins share two common features which are a cationic region (the so-called "active region") and hydrophobic patch. Both a- and $\beta$-defensins have three disulfide bonds (Cys1-Cys6, Cys2-Cys4, Cys3Cys5 in a-defensins and Cys1-Cys5, Cys2-Cys4, Cys3-Cys6 in $\beta$-defensins) and three-stranded $\beta$-sheet structures ( $\beta 1$ - $\beta 3$ in Fig. 1A), whereas $\theta$-defensins form cyclic structures [4]. In humans, six human $\alpha$ defensins are identified which are human neutrophil peptides 1 to 4 (HNP1-HNP4) and human enteric defensins 5 (HD5) and 6 (HD6) [5]. HNP1-HNP4 are found in neutrophil granules, whereas HD5 and HD6 are expressed in Paneth cells [6].

Among six a-defensins, HD5 was reported to show a high level of activities against gram-negative and gram-positive bacteria [7]. It is a cationic and amphipathic peptide comprising 32 residues with a total charge of +4 at neutral $\mathrm{pH}[6,8]$. HD 5 contains three $\beta$-strands ( $\beta 1$ - $\beta 3$ ) linking by coil (T1) and turn (T2) (Fig. 1) with cationic and amphipathic characters. One of possible bacteria-killing mechanisms of HD5 is to disrupt a membrane by making a pore and penetrate into a cytoplasm [9]. Different oligomeric states were identified with different degrees of antimicrobial activity and structural stability $[10,11]$. In solution, a dimer was found to be the most active oligomer, while the largest stable form is a tetramer $[11,12,13]$. Moreover, HD5 also shows the ability to neutralise bacterial lipopolysaccharide (LPS) [14, 15]. Many experimental studies have attempted to understand the effects of HD5-LPS binding on microbial cell activity $[9,15,16]$, but the microscopic information remains unclear. Recently, the adsorption mechanism of HD5 on a LPS membrane surface has been computationally revealed [8], however neither protein penetration nor pore formation was observed. Recent studies show the ability of HD5 to enter microbial 
and viral cytoplasm $[9,17,18,19]$, but a mechanism of intracellular translocation of HD5 into a cytoplasm is not well-defined. Thus, in this work, the mechanism of pore formation by a dimeric HD5 through a gram-negative LPS membrane is explored in atomic level using Steered Molecular dynamics simulation (SMD). Only simplified LPS model (Re-LPS) is used because of the LPS complexity.

\section{Materials And Methods}

The starting HD5-LPS complex here was obtained from a previous work where a system preparation can be seen in [8]. The final snapshot of a dimeric HD5-LPS complex from a previous work was used. A simplified LPS (the inner core region (Re-LPS) composing of lipid A and 3-deoxy-d-manno-octulosonic acid (KDO)) was employed here where LPS parameters were obtained from a previous work [20]. Counter ions are then added to neutralise a system. All systems were energy minimized for 10,000 steps to remove bad contacts. To explore the penetration of HD5 into a LPS membrane, the constant-velocity SMD simulations were performed for $200 \mathrm{~ns}$. An external force was applied in the z-direction (perpendicular to a membrane axis) by attaching a virtual harmonic spring with a force constant of $1,000 \mathrm{~kJ} \cdot \mathrm{mol}^{-1} \cdot \mathrm{nm}^{-2}$ to the center of mass of a dimeric HD5 protein. The direction of pulling is from the outer leaflet to the inner leaflet ( $Z \diamond-Z$ direction) with the rate of $0.04 \mathrm{~nm} / \mathrm{ns}$. Two replicates were performed with different random seeds. "HD5_1" and "HD5_2" are used to represent the first and the second simulations.

All reported simulations were performed using the GROMACS 5.0 package with GROMOS 53A6 forcefields [21]. The particle mesh Ewald (PME) method was used for electrostatic treatment with a shortrange cut-off of $1 \mathrm{~nm}$ and a Fourier spacing of $0.12 \mathrm{~nm}$. A 2 fs integration time step was used with LINCS algorithms. The simulations were conducted in NPT ensemble using semi-isotropic Parrinello-Rahman barostat with $\tau_{p}=1 p s$ and v-rescale thermostat at $323 \mathrm{~K}$ [22] with a coupling constant of $\tau_{p}=0.1 p s$ where protein, membrane, and solution were coupled separately.

All outcomes were analysed by GROMACS tools and in-house codes. The hydrogen bonds were computed using g_hbond with default parameters (The hydrogen-donor-acceptor cutoff angle is $30^{\circ}$ and the cutoff radius (X-acceptor) as $0.35 \mathrm{~nm}$ ). VMD was used for visualization and preparing molecular graphic images [23].

\section{Results And Discussions}

At the beginning, the positions of centre of mass of a dimeric HD5 along a permeation path $(+Z \diamond-Z)$ and exerted forces on HD5 are calculated and shown in Fig. 2A and 2B. For both simulations, the double force wells are observed from $Z \sim 0-2 \mathrm{~nm}$ whose positions are in the outer leaflet (Fig. 2A). Two exerted force peaks (a major peak at $Z \sim 2 \mathrm{~nm}(\mathrm{~F} 1)$ and a minor one at $\mathrm{Z} \sim 0-1 \mathrm{~nm}(\mathrm{~F} 2)$ ) are derived from the strong electrostatic interactions between HD5 and lipid A head groups (KDO and Pi) (Fig. 2A). Then, HD5_1 and HD5_2 arrive a hydrophobic core at $100 \mathrm{~ns}$ and $136 \mathrm{~ns}$, corresponding to $\mathrm{Z}=0 \mathrm{~nm}$ for HD5_1 and $-1 \mathrm{~nm}$ for HD5_2. These different $Z$ positions observed are due to the curvature of LPS membrane (Fig. $2 C$ ). Besides, a drop of $Z$ positions before $100 \mathrm{~ns}$ for HD5_1 ( $Z$ from $1 \diamond 0 \mathrm{~nm}$ ) and $136 \mathrm{~ns}$ for HD5_2 (Z from 
$0 \diamond-1 \mathrm{~nm}$ ) indicates a quick move from the outermost layer into a membrane core (Fig. 2B). This drop suggests the less afford required to dive into a centre of hydrophobic core. To pass through the inner leaflet ( $Z$ from $0 \diamond \sim-4$ for HD5_1 and Z from $-1 \diamond-4$ for HD5_2), two comparable sizes of exerted forces are observed (F3 and F4 in Fig. 2A). These two applied forces refer to the barriers for dragging HD5 out of a hydrophobic core (F3) and phospholipid head groups (F4). The force profiles clearly show that passing through a LPS surface into a membrane core requires much larger exerted force than going out of the inner leaflet due to strong charge-charge interactions between HD5 and lipid A head groups. Seemingly, the electrostatic interactions between HD5 and lipid A head groups cause a charged LPS surface to be a facilitator for the HD5 adhesion, but hindrance for the passage of HD5 at the same time. In addition, the HD5 translocation causes a water leakage in both simulations (Fig. 3B). As seen earlier, the curvature of a LPS membrane is found in both simulations, although HD5_2 shows a higher degree of a membrane curvature. A pulling force seems to influence a LPS membrane in HD5_2, however both HD5_1 and HD5_2 can provide a similar pattern of force profiles (Fig. 2A). This implies our SMD work can be repeated. Moreover, in Fig. 2C, HD5 in both simulations employ only one chain to adhere on a LPS membrane. In this work, only chains $b$ is used to stick on a host membrane.

Comparing to X-ray structure, the protein conformation of HD5_1 seems to be more preserved than HD5_2. Overall, the conformational change of T1 in chain $b$ in all cases is observed (Fig. 2C). T1 region is thus the most flexible part where higher T1 flexibility is found in HD5_2 (Fig. 2C and S1 in supplementary information). T1 in chain $b$ starts to move away from its $\beta$-stranded core region in both simulations, while this move allows the dimer dissociation at the end of simulation in HD5_2 (Fig. 2C). Such T1 dynamics may be important to facilitate the HD5 adsorption and permeation because this T1 reorientation is preserved throughout an additional conventional MD work (unpublished work). To observe how HD5 passage affects the outer leaflet, the density maps of LPS membrane are computed in Fig. 3A. It is clear in Fig. $3 \mathrm{~A}$ that a dimeric HD5 interrupts the distribution of polar moietires on a LPS surface which can destabilise the outer leaflet. Different sizes of water-filled pores generated by each simulation are shown in Fig. 3A (green circles). Different pore sizes are formed due to the different alignments of a HD5 dimer inside a membrane (Fig. 3B). Although both systems employ chain $b$ to land on a membrane interface, inside a membrane, HD5_1 seems to align perpendicularly to the $z$ axis, while HD5_2 is in parallel (Fig. 3B) which causes various degrees of water leakage during a HD5 travel.

Considering the HD5-LPS interactions, a dimeric HD5 strongly interacts with both KDO sugars and phosphate (Pi) moieties of lipid A, but the higher number of KDO interactions are found (Fig. 3C). Upon arrival, a high number of HD5-KDO interactions is formed ( 15 hydrogen bonds), but they are dropped when HD5 translocates close to a hydrophobic region (after $75 \mathrm{~ns}$ ). Nonetheless, a loss of such hydrogen bonds is compensated by HD5-Pi (outer) interactions (Fig. 3C). As expected, HD5 gains more hydrogen bonds with $\mathrm{Pi}$ (inner) of phospholipid head groups when it travels close to a surface of the inner leaflet (Fig. 3C). Apparently, a dimeric HD5 is continuously wrapped by polar groups of lipid A along its permeation pathway. It means that the lipid A head groups are dragged down into a membrane core by a cationic HD5. During a HD5 insertion, the lipid A head groups (KDO and $\mathrm{Pi}$ ) are not only pulled down into a membrane core, but they also carry water molecules with them. KDO sugars and Pi are found to escort 
a comparable number of water molecules down into a membrane centre (Figure $\mathrm{S} 2$ in supplementary information). Besides, a presence of constant KDO- and $\mathrm{Pi}$ (outer)- water contacts suggests the existence of a water-filled pore (Figure S2 in supplementary information). Altogether, both pulled lipid $A$ head groups and permeated HD5 induce the entry of water into a hydrophobic region resulting in a membrane disruption and water leakage along a membrane axis. This highlights a key role of electrostatic interactions between a cationic HD5 and LPS surface on the pore-forming activity.

To further investigate the behaviour of a HD5 dimer, sets of distances between two monomers and a pair of key residues (V19-E21) for dimerization are measured in Fig. 4A and 4B. In HD5_2, The shifted distances between two chains (chains $a$ and $b$ ) and V19-E21 at 136 ns obviously illustrates the dimer separation (Fig. 4A and 4B). A V19-E21 interaction was reported to be important for dimerization [8, 11, 24]. Thus, the breakdown of V19-E21 pair in HD5_2 serves as a sign for a dimer dissociation (Fig. 4B). The orientations of V19 and E21 can be seen in Fig. 4C. In contrast, a dimeric HD5_1 is more stable owing to the ability to maintain a V19-E21 interaction (Fig. 4B and 4C). In addition, hydrogen bonds of HD5membrane and HD5-water are also computed. HD5_1 and HD5_2 can form interactions with both membrane and water along a permeation path (Fig. 4D and 4E). This confirms the ability of a dimeric HD5 to drag polar moieties of lipid A and water molecules down into a membrane core. In Fig. 4D, a number of HD5-membrane hydrogen bonds are gradually increased until $~ 75 \mathrm{~ns}$ due to a close contact to a hydrophilic membrane surface and then reduced after $100 \mathrm{~ns}$ when approaching a hydrophobic region. A sharp peak of HD5-water interactions is captured at $136 \mathrm{~ns}$ in HD5_2. This is because, at 136 ns, the breakdown of HD 5 causes a sudden loss of contacts with KDO and Pi at the outer leaflet which requires more water interactions to compensate (Fig. $3 \mathrm{C}$ and $4 \mathrm{E}$ ). Nonetheless, after $136 \mathrm{~ns}$, a number of water contacts drop down since HD5 gains more Pi(inner) contacts from the inner leaflet (Fig. 3C and 4E).

In addition, sets of hydrogen bonds between each chain and its environment are investigated (Fig. 5). Because a dimeric HD5 utilises chain $b$ to anchor on a LPS membrane, a high number of chain $b$ membrane hydrogen bonds are captured ( 15 hydrogen bonds in Fig. $5 B)$. Only $~ 7.5$ chain a-membrane interactions are found (Fig. 5A). However, a comparable degree of water interactions is observed in both chains. Expectedly, the water contacts are maximized at the membrane surface in both simulations (Fig. 5C and 5D). Continuous interactions with water in Fig. 5C and 5D indicate a presence of water-filled pore. A sudden shift of chain a-water hydrogen bonds at Z -1 nm in HD5_2 corresponding to a dimer separation as explained earlier (Fig. 5C).

For further analysis, the residue-membrane hydrogen bonds are computed (Fig. 6). Upon binding, chain $b$ of a dimeric HD5 employs its T1 (residue 6-14) and $\beta 3$ (residue 25-32) regions to interact with a membrane surface via strong interactions with arginines in the active region ( $R 6, R 9, R 13, R 25, R 28$, and R32) (Figs. 1, 2 and 6). Not only arginines, but other polar residues also contribute to the adsorption. In case of chain $a$, it can interact with a membrane using R13, R32, A1, and T2 (Fig. 6). Seemingly, HD5 requires one main chain to stick on a cell surface, whereas the other chain acts as an assistant. When HD 5 approaches the inner leaflet, an additional set of interactions are formed (after $100 \mathrm{~ns}$ ). A strand $\beta 3$ of chain $a$ in both simulations flips down and align normal to a membrane axis and forms hydrogen 
bonds with $\mathrm{C} 3, \mathrm{~S} 23, \mathrm{R} 25, \mathrm{Y} 27, \mathrm{R} 28$ (Fig. 6). In HD5_1, chain $b$ employs a part of $\beta 2$ to additionally interact with a membrane (L16, S17, G18, I22), while the extra interactions with $A 1$ and $C 5$ on $\beta 1$ are found in HD5_2 (Fig. 6). In chain $b$, the conformational change of T1 region is found (Fig. 2C). This region contains three arginines (R6, R9, and R13) which form strong interactions with charged moieties of lipid A (Fig. 6). Such interactions may trap T1 and cause the change in T1 orientation. The effect of lipid interactions on a defensin conformation is also seen in other a -defensins $[25,26]$. Unlike chain $a$ in HD5_1, more residue-membrane hydrogen bonds are found in chain a of HD5_2 (Fig. 6). Such interactions allow the tighter binding of chain a to lipid A layer which can retard a HD5 passage and lead to the split of a dimer in HD5_2.

Overall, our results show that all arginines in the active region contribute to charge-charge interactions between HD5 and a LPS surface. Persistent arginine-membrane hydrogen bonds observed in Fig. 6 demonstrate the role of arginine residues in both adsorption and permeation. Especially, R13 and R32 of both chains in all simulations are found to form interactions with a membrane (Fig. 6). This implies the important role of both R13 and R32 in HD5's activity. The double mutation of R13 and R32 to alanine was previously reported to reduce the antibacterial activity against gram-negative bacteria [27]. Based on our results, a loss of R13 and R32 contacts can diminish the binding affinity of HD5 to host cell surface resulting in a decrease in antibacterial activity as seen in a previous work. Besides, R9 and R28 were reported to be important for killing gram-positive strains, especially $B$. cereus [27]. In our case, insignificant interactions with R9 and R28 are observed (Fig. 6A). This demonstrates a minor role of R9 and R28 in interacting with our gram-negative membrane model. Comparing among six defensins, HD5 contains the highest number of arginines (Figure S3 in supplementary information). This may be one of factors that allow HD 5 to show high level of bacteria-killing activity [7]. Arginine residues appear to be crucial for interacting with LPS head groups which facilitate a HD5 adhesion and disrupting a membrane structure. This finding can be used to explain why more arginines can enhance HD5 activity as seen in a previous work [28]. Seemingly, the HD5 function is dependent on its cationicity. However, the substitution of arginine to lysine was found to disturb the bacterial killing ability of HD5 [27, 29]. For example, the double mutation of arginine to lysine (R1332K and R928K) causes the reduction of E.coli killing, but only R928K affects the bacterial activity against S.aureus [27]. It appears that the arginine-to-lysine substitution affects the bacterial killing activity selectively. Although it was reported that the different degrees of hydrophobicity and ability for electrostatic interactions between arginine and lysine cause the selective antibacterial activity [27, 30,31], the understanding of how lysine influences the antibacterial activity remains unclear.

\section{Conclusions}

In this work, the penetration of a dimeric HD5 through a LPS membrane is investigated. It is found in this work that three key barriers for HD5 passage are (i) negatively charged LPS surface (KDO and Pi of lipid A) of the outer leaflet, (ii) hydrophobic core region, and (iii) phospholipid head groups (Pi layer). The strong electrostatic interactions between HD5 and LPS membrane appear to serve as a facilitator for HD5 adsorption and a major barrier for HD5 permeation at the same time. Arginine residues are the main 
player for HD5-membrane electrostatic interactions, whereas the hydrophobic interactions become minor. When approaching a LPS interface, HD5 employs one chain to adhere on a membrane surface. Arginines are used to interact with lipid A head groups. During a HD5 insertion, HD5 drags both lipid A head groups and water molecules down into a hydrophobic core. This event does not only destabilise a membrane, but also induce a water leakage leading to the formation of water-filled pore. To date, it is not clear how HD5 interferes a bacterial cell surface because the effects on each bacterium are different. Also, it has been recently discovered that HD5 contributes to the pathogenicity of Shigella, HIV, and SARS-CoV2 by mediating the adhesion of these pathogens to target cells $[19,32,33,34,35,36]$. HD 5 can either kill bacteria or support their infection depending on the complexity of host interactions at interfaces. In this work, it is clear that interactions with lipid A head groups are the key for successful HD5 attachment on a host cell surface. However, an information on specificity and selectivity of HD5 on host cell surface remains unclear and needs to be understood for development of new strategies for microbial prevention.

\section{Abbreviations}

MD

Molecular dynamics

SMD

Steered molecular dynamics

LPS

Lipopolysaccharide

HD5

Human a-defensin 5

KDO

3-deoxy-d-manno-octulosonic acid

\section{Declarations}

\section{Acknowledgements}

We would like to thank Kasetsart University Research and Development Institute (KURDI, grant no. FF(KU) 25.64), Faculty of Science, Department of Chemistry (BRF policy) and Science Achievement Scholarship of Thailand (SAST) for financial support.

\section{Funding}

This work is supported by Kasetsart University Research and Development Institute (KURDI, grant no. FF(KU) 25.64), Faculty of Science, Department of Chemistry (BRF policy) and Science Achievement Scholarship of Thailand (SAST) for financial support.

\section{Conflict of intertest}


This manuscript is original, has not been published before and is not currently being considered for publication elsewhere. There are no known conflicts of interest associated with this publication and there has been no significant financial support for this work that could have influenced its outcome.

\section{Availability of data and material: N/A}

Code availability: N/A

Authors' contributions: TA conducted the simulations, analysed data, and wrote a manuscript. PP analysed data and wrote a manuscript.

\section{References}

1. Jung SW, Lee J, Cho AE (2017) Elucidating the Bacterial Membrane Disruption Mechanism of Human alpha-Defensin 5: A Theoretical Study. J Phys Chem B 121:741-748

2. Huan Y, Kong Q, Mou H, Yi H (2020) Antimicrobial Peptides: Classification, Design, Application and Research Progress in Multiple Fields. Front Microbiol 11:582779. Epub 2020/11/13

3. Zhang L (2017) Different dynamics and pathway of disulfide bonds reduction of two human defensins, a molecular dynamics simulation study. Proteins: Struct Funct Bioinf 85:665-681

4. Selsted ME, Ouellette AJ (2005) Mammalian defensins in the antimicrobial immune response. Nature immunology 6:551

5. Lehrer RI, Lu W (2012) alpha-Defensins in human innate immunity. Immunol Rev 245:84-112. Epub 2011/12/16

6. Rajabi M, Ericksen B, Wu XJ, de Leeuw E, Zhao L, Pazgier M, Lu WY (2012) Functional Determinants of Human Enteric alpha-Defensin HD5 CRUCIAL ROLE FOR HYDROPHOBICITY AT DIMER INTERFACE. J Biol Chem 287:21615-21627

7. Ericksen B, Wu Z, Lu W, Lehrer RI (2005) Antibacterial activity and specificity of the six human \{alpha\}-defensins. Antimicrob Agents Chemother 49:269-275

8. Awang T, Pongprayoon P (2018) The adsorption of human defensin 5 on bacterial membranes: simulation studies. J Mol Model 24:273. Epub 2018/09/07

9. Chileveru HR, Lim SA, Chairatana P, Wommack AJ, Chiang IL, Nolan EM (2015) Visualizing attack of Escherichia coli by the antimicrobial peptide human defensin 5. Biochemistry-Us 54:1767-1777

10. Nguyen LT, Haney EF, Vogel HJ (2011) The expanding scope of antimicrobial peptide structures and their modes of action. Trends in biotechnology 29:464-472

11. Chairatana P, Niramitranon J, Pongprayoon P (2019) Dynamics of human defensin 5 (HD5) selfassembly in solution: Molecular simulations/insights. Comput Biol Chem 83:107091. Epub $2019 / 07 / 28$

12. Wommack AJ, Robson SA, Wanniarachchi YA, Wan A, Turner CJ, Wagner G, Nolan EM (2012) NMR solution structure and condition-dependent oligomerization of the antimicrobial peptide human 
defensin 5. Biochemistry-Us 51:9624-9637

13. Lehrer RI, Jung G, Ruchala P, Andre S, Gabius HJ, Lu W (2009) Multivalent binding of carbohydrates by the human alpha-defensin, HD5. J Immunol 183:480-490

14. Chen F, Tang Y, Zheng H, Xu Y, Wang J, Wang C (2019) Roles of the Conserved Amino Acid Residues in Reduced Human Defensin 5: Cysteine and Arginine Are Indispensable for Its Antibacterial Action and LPS Neutralization. ChemMedChem 14:1457-1465. Epub 2019/07/11

15. Wang C, Shen M, Zhang N, Wang S, Xu Y, Chen S, Chen F, Yang K, He T, Wang A, Su Y, Cheng T, Zhao J, Wang J (2016) Reduction Impairs the Antibacterial Activity but Benefits the LPS Neutralization Ability of Human Enteric Defensin 5. Sci Rep 6:22875

16. Scott MG, Vreugdenhil AC, Buurman WA, Hancock RE, Gold MR (2000) Cutting edge: cationic antimicrobial peptides block the binding of lipopolysaccharide (LPS) to LPS binding protein. J Immunol 164:549-553. Epub 2000/01/07

17. Mathew B, Nagaraj R (2015) Antimicrobial activity of human alpha-defensin 5 and its linear analogs: $\mathrm{N}$-terminal fatty acylation results in enhanced antimicrobial activity of the linear analogs. Peptides 71:128-140

18. Mathew B, Nagaraj R (2017) Variations in the interaction of human defensins with Escherichia coli: Possible implications in bacterial killing. PLoS One 12:e0175858. Epub 2017/04/20

19. Xu D, Lu W. Defensins (2020) A Double-Edged Sword in Host Immunity. Front Immunol 11:764. Epub 2020/05/28

20. Hsu P-C, Jefferies D, Khalid S (2016) Molecular Dynamics Simulations Predict the Pathways via Which Pristine Fullerenes Penetrate Bacterial Membranes. J Phys Chem B 120:11170-11179

21. Oostenbrink C, Villa A, Mark AE, Gunsteren WFV (2004) A biomolecular force field based on the free enthalpy of hydration and solvation: The GROMOS force-field parameter sets $53 A 5$ and 53A6. J Comput Chem 25:1656-1676

22. Jung SW, Lee J, Cho AE (2017) Elucidating the Bacterial Membrane Disruption Mechanism of Human a-Defensin 5: A Theoretical Study. J Phys Chem B 121:741-748

23. Humphrey W, Dalke A, Schulten K (1996) VMD: Visual molecular dynamics. J Mol Graph 14:33-38

24. Szyk A, Wu ZB, Tucker K, Yang D, Lu WY, Lubkowski J (2006) Crystal structures of human alphadefensins HNP4, HD5, and HD6. Protein Sci 15:2749-2760

25. Zhang Y, Lu W, Hong M (2010) The membrane-bound structure and topology of a human alphadefensin indicate a dimer pore mechanism for membrane disruption. Biochemistry-Us 49:97709782. Epub 2010/10/22

26. Yeasmin R, Buck M, Weinberg A, Zhang L (2018) Translocation of Human beta Defensin Type 3 through a Neutrally Charged Lipid Membrane: A Free Energy Study. J Phys Chem B 122:1188311894. Epub 2018/11/16

27. de Leeuw E, Rajabi M, Zou G, Pazgier M, Lu W (2009) Selective arginines are important for the antibacterial activity and host cell interaction of human alpha-defensin 5. FEBS Lett 583:2507-2512. 
Epub 2009/07/11

28. Wang C, Zhao G, Wang S, Chen Y, Gong Y, Chen S, Xu Y, Hu M, Wang X, Zeng H, Wang A, Liu D, Su Y, Cheng T, Chen F, Wang J. A Simplified Derivative of Human Defensin 5 with Potent and Efficient Activity against Multidrug-Resistant Acinetobacter baumannii. Antimicrob Agents Chemother. 2018;62. Epub 2017/11/22

29. Zou GZ, de Leeuw E, Li C, Pazgier M, Li CQ, Zeng PY, Lu WY, Lubkowski J, Lu WY (2007) Toward understanding the cationicity of defensins - Arg and Lys versus their noncoded analogs. $\mathrm{J}$ Biol Chem 282:19653-19665

30. Nozaki Y, Tanford C (1971) The solubility of amino acids and two glycine peptides in aqueous ethanol and dioxane solutions. Establishment of a hydrophobicity scale. J Biol Chem 246:22112217. Epub 1971/04/10

31. Dougherty DA (1996) Cation-pi interactions in chemistry and biology: a new view of benzene, Phe, Tyr, and Trp. Science 271:163-168. Epub 1996/01/12

32. Liao C, Fang K, Xiao J, Zhang W, Zhang B, Yuan W, Lu W, Xu D (2019) Critical determinants of human neutrophil peptide 1 for enhancing host epithelial adhesion of Shigella flexneri. Cell Microbiol 21:e13069. Epub 2019/06/21

33. Xu D, Liao C, Xiao J, Fang K, Zhang W, Yuan W, Lu W. Human Enteric Defensin 5 Promotes Shigella Infection of Macrophages. Infect Immun. 2019;88. Epub 2019/10/16

34. Xu D, Liao C, Zhang B, Tolbert WD, He W, Dai Z, Zhang W, Yuan W, Pazgier M, Liu J, Yu J, Sansonetti PJ, Bevins CL, Shao Y, Lu W. Human Enteric alpha-Defensin 5 Promotes Shigella Infection by Enhancing Bacterial Adhesion and Invasion. Immunity. 2018;48:1233-44 e6. Epub 2018/06/03

35. Rapista A, Ding J, Benito B, Lo YT, Neiditch MB, Lu W, Chang TL (2011) Human defensins 5 and 6 enhance HIV-1 infectivity through promoting HIV attachment. Retrovirology 8:45. Epub 2011/06/16

36. Niv Y (2020) Defensin 5 for prevention of SARS-CoV-2 invasion and Covid-19 disease. Med Hypotheses 143:110244. Epub 2020/10/07

\section{Figures}



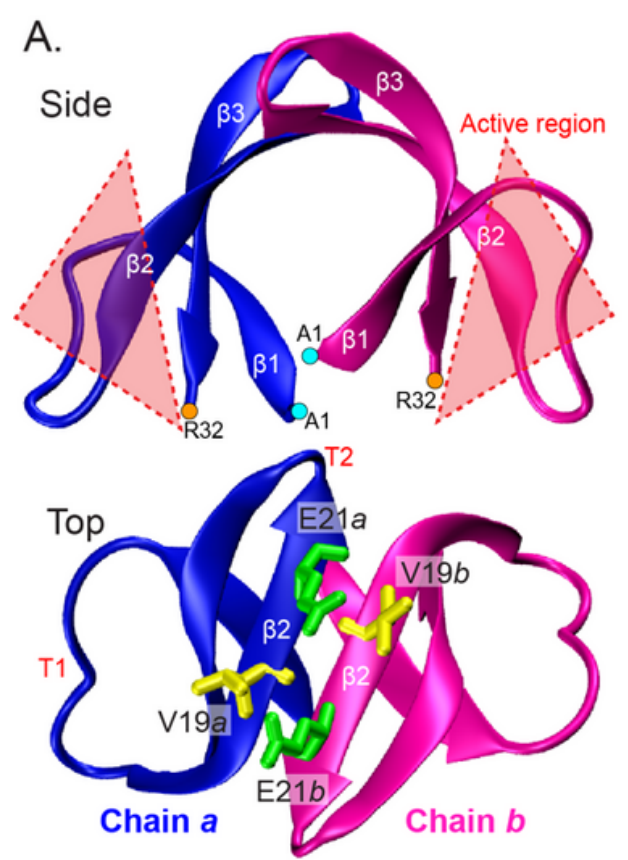

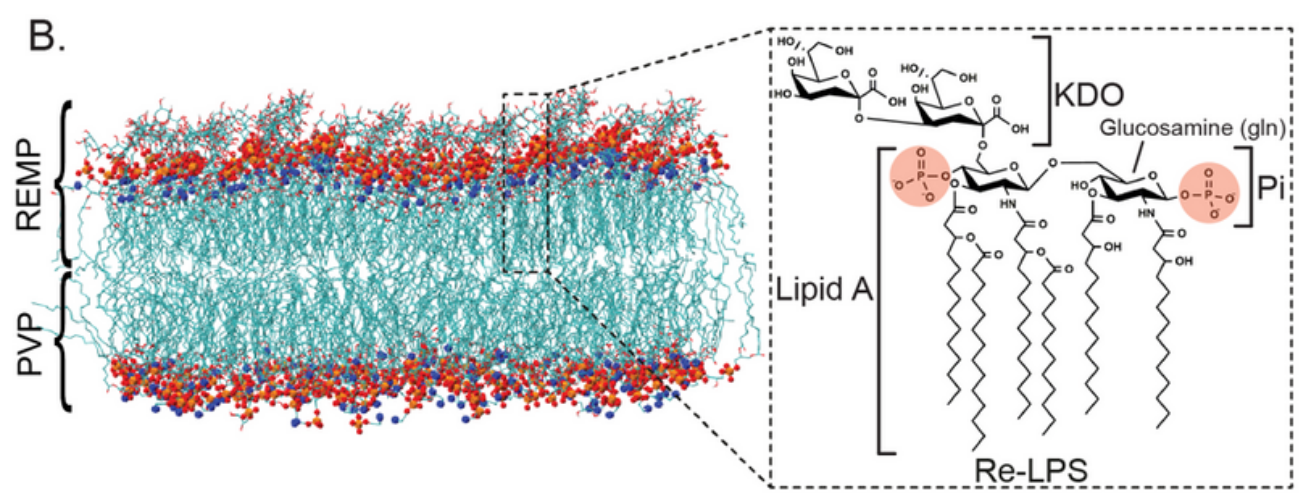

C.

(II) Cys2-Cys4

\section{HD5}

(I) Cys1-Cys6

(III) Cys3-Cys5
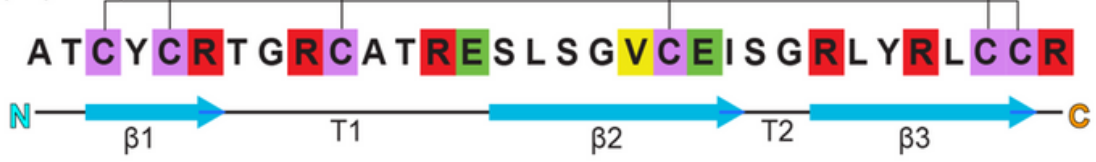

Figure 1

(A) Cartoon view of dimeric HD5 consisting of chains a and $b$. The arginine-rich regions (the active region) are shown in the red dashed triangle, whereas key residues (V19 and E21) for dimerization are shown in licorice format. (B) Lipopolysaccharide (LPS) membrane. Its chemical structure is shown in an inset. Only 3-deoxy-d-manno-octulosonic acid (KDO) and Lipid A are involved in this LPS membrane model. (C) Amino acid sequences of HD5. Conserved cysteines are shown in purple. Valine is in yellow. Positively charged and negatively charged residues are in red and green, respectively. 

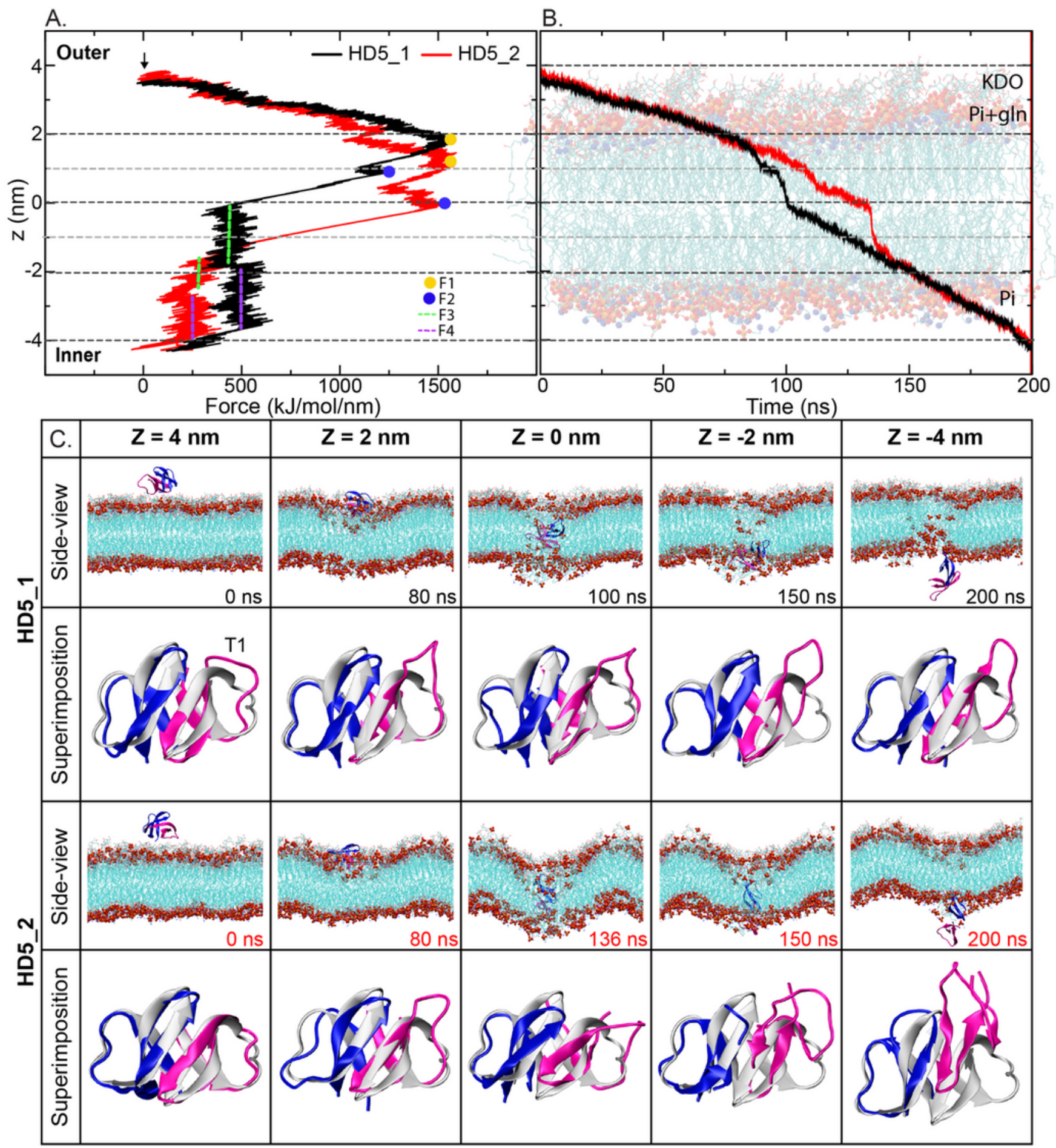

Figure 2

HD5 positions along a membrane axis (z-axis) as functions of exerted force $(A)$ and time (B). Layers of each composition (KDO, phosphate region (Pi), lipid tails) are also shown. (C) shows the positions of a dimeric HD 5 and superimposition between X-ray structure (grey) and a dimeric HD5 at z = 4, 2, 0, -2, and $-4 \mathrm{~nm}$, respectively. Chains $\mathrm{a}$ and $\mathrm{b}$ are labelled in blue and pink. 


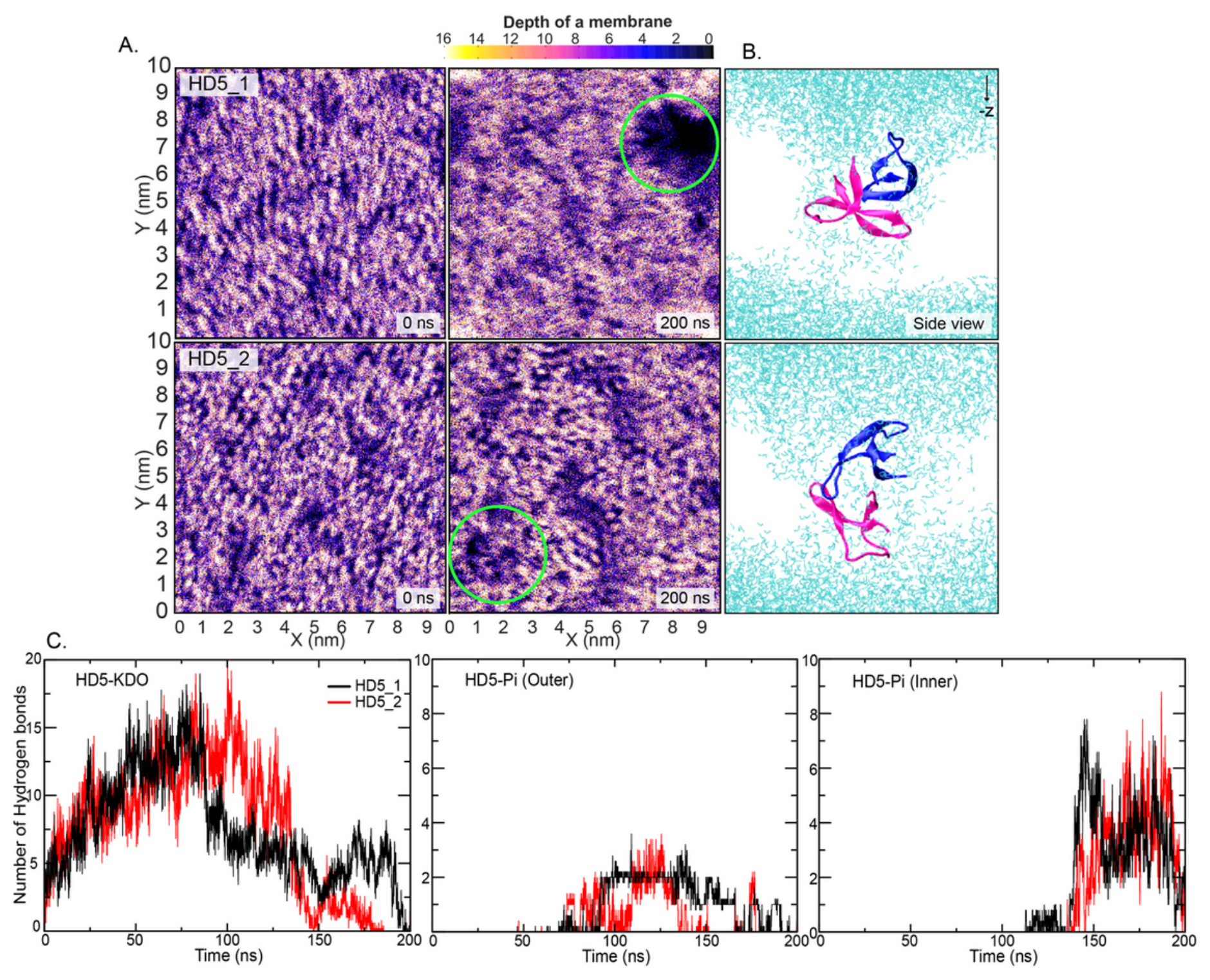

Figure 3

Density map of the LPS outer leaflet in XY plane of all systems at 0 ns and 200 ns (A). Green circle indicates the HD5 site. (B) Orientations of a dimeric HD5 at the mid of a bilayer in each system. (C) Hydrogen bonds of HD5-KDO, HD5-Pi (outer), and HD5-Pi (inner) where "outer" and "inner" stand for the outer and inner leaflets. 

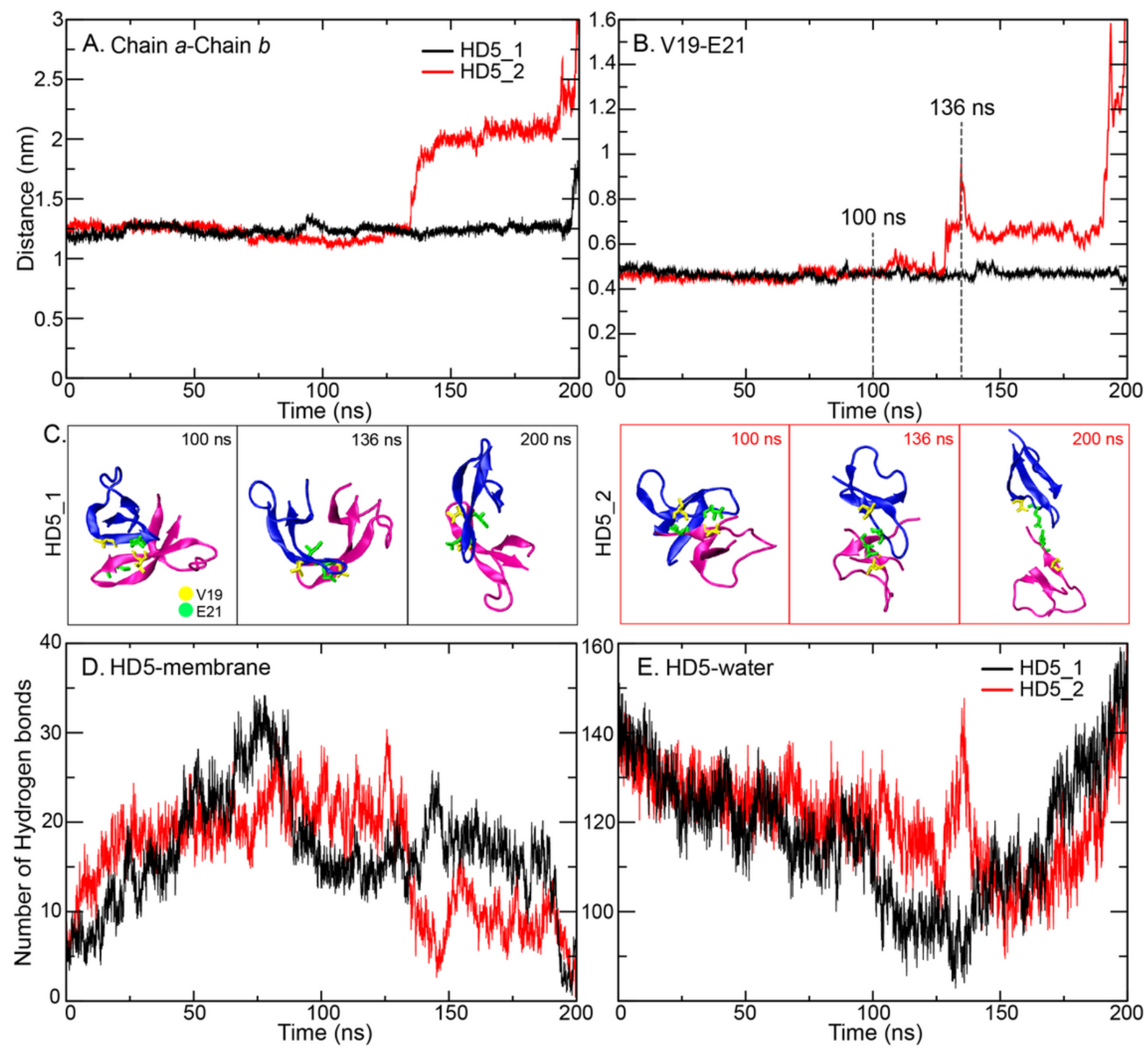

Figure 4

Distances between centre of masses (COM) of chains a and b (A) and V19-E21 (B) as a function of time. (C) illustrates the orientation of dimeric HD5 in HD5_1 and HD5_2 at time 100 ns, 136 ns, and 200 ns. V19 and E21 are labelled in yellow and green. (D)-(E) are HD5-membrane and HD5-water hydrogen bonds in all simulations. 


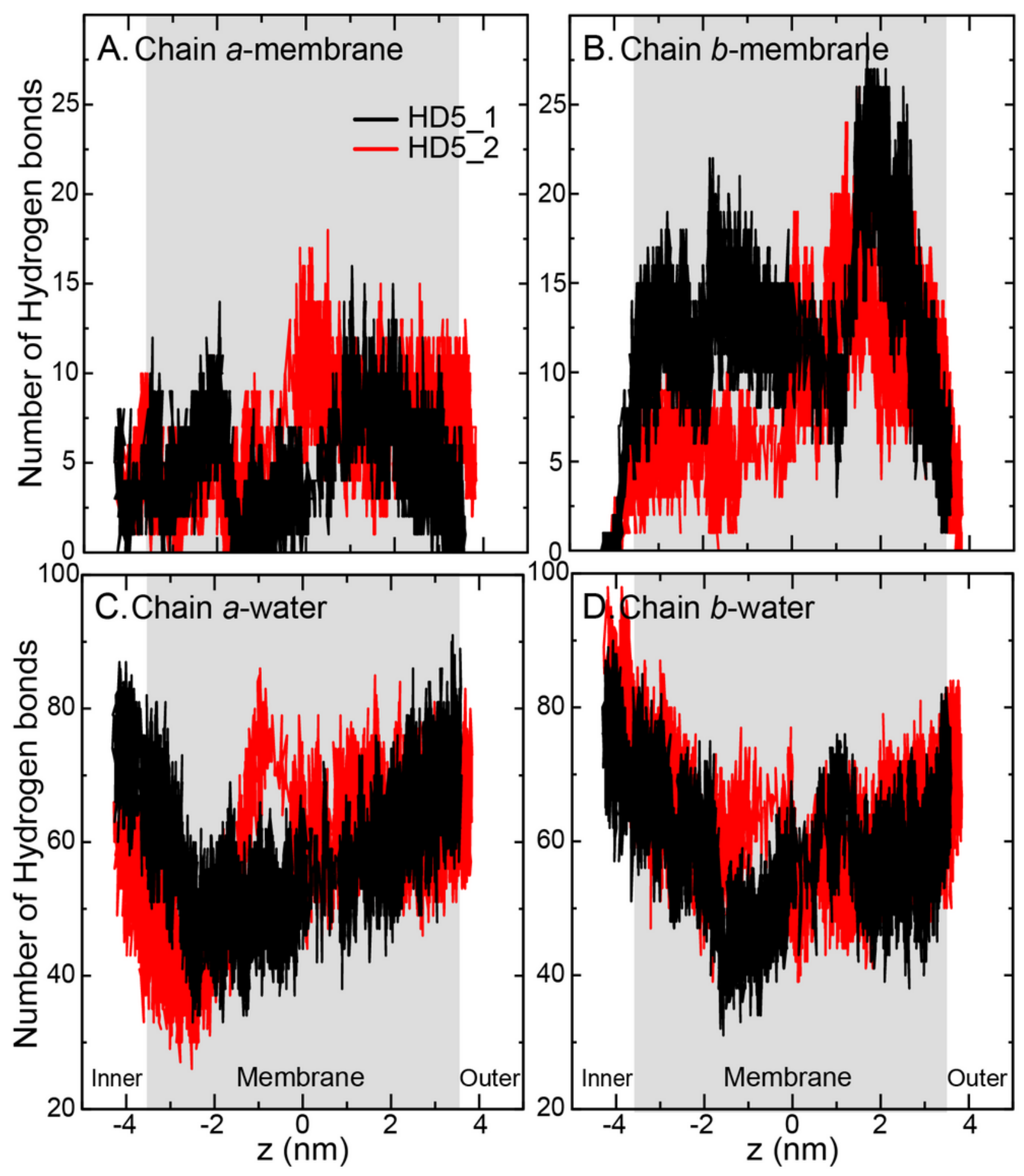

Figure 5

A number of hydrogen bonds as a function of coordinates between each chain and membrane (A)-(B) and water (C)-(D). Grey bands indicate a membrane region. 


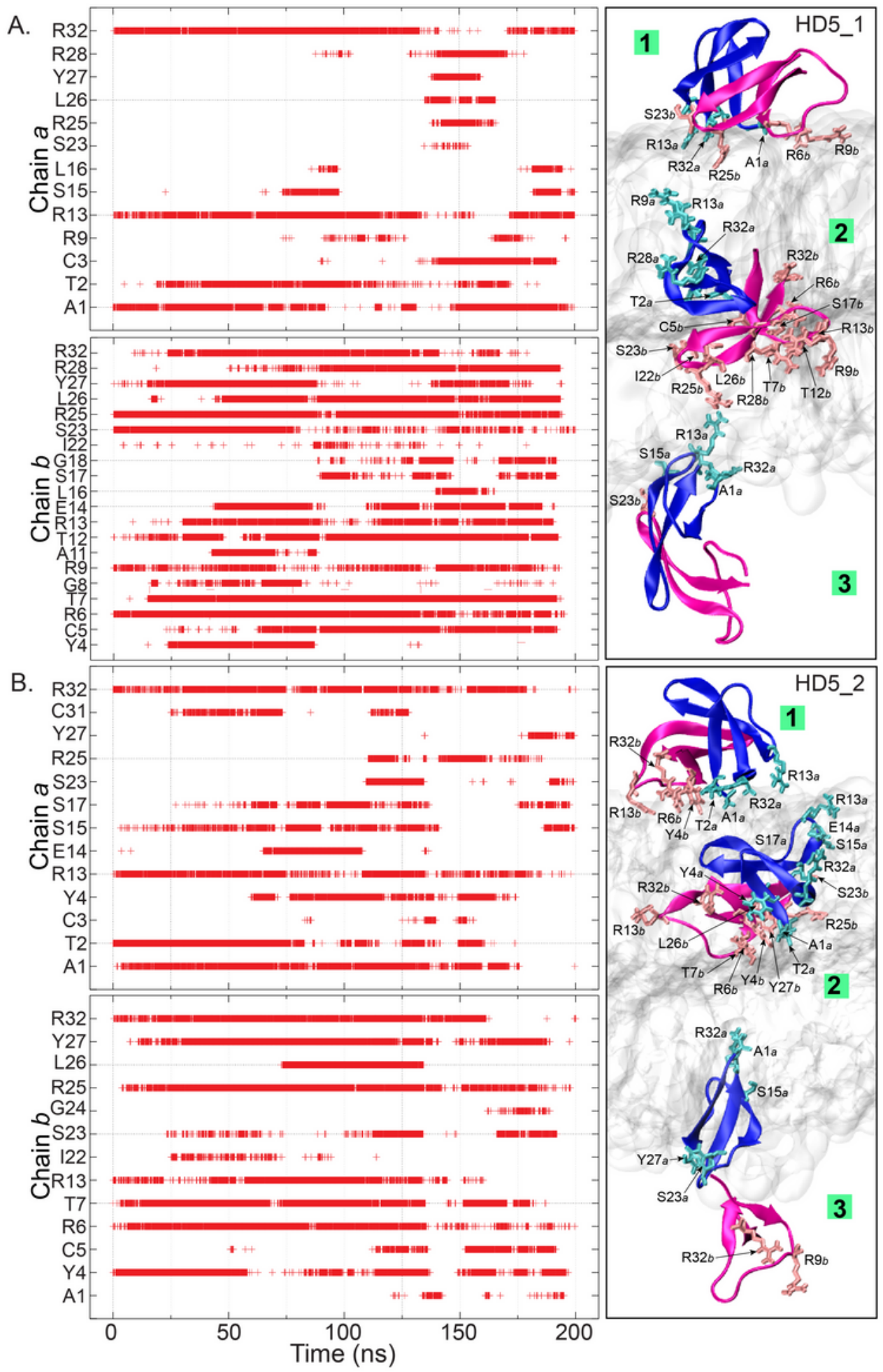

\section{Figure 6}

Hydrogen bonds between key residues from each chain and a LPS membrane as a function of time for HD5_1 (A) and HD5_2 (B). The protein orientations (blue and pink represent chains a and $b$ ) on a membrane (a gray layer) and key residues at time 0 ns ("1"), 100 ns ("2"), and 200 ns (" 3 ") are displayed on the right. 


\section{Supplementary Files}

This is a list of supplementary files associated with this preprint. Click to download.

- graphab.tif

- SIHD5SMDlatest.docx 